\title{
Hierarchical Construction of Higher-Order Exceptional Points
}

\author{
Q. Zhong $\odot,{ }^{1,2}$ J. Kou, ${ }^{3}$ Ş. K. Özdemir®, ${ }^{4}$ and R. El-Ganainy ${ }^{1,2, *}$ \\ ${ }^{1}$ Department of Physics, Michigan Technological University, Houghton, Michigan 49931, USA \\ ${ }^{2}$ Henes Center for Quantum Phenomena, Michigan Technological University, Houghton, Michigan 49931, USA \\ ${ }^{3}$ Division of Engineering and Applied Science, California Institute of Technology, Pasadena, California 91125, USA \\ ${ }^{4}$ Department of Engineering Science and Mechanics, and Materials Research Institute, The Pennsylvania State University, \\ University Park, Pennsylvania 16802, USA
}

(Received 18 March 2020; accepted 28 September 2020; published 12 November 2020)

\begin{abstract}
The realization of higher-order exceptional points (HOEPs) can lead to orders of magnitude enhancement in light-matter interactions beyond the current fundamental limits. Unfortunately, implementing HOEPs in the existing schemes is a rather difficult task, due to the complexity and sensitivity to fabrication imperfections. Here we introduce a hierarchical approach for engineering photonic structures having HOEPs that are easier to build and more resilient to experimental uncertainties. We demonstrate our technique by an example that involves parity-time symmetric optical microring resonators with chiral coupling among the internal optical modes of each resonator. Interestingly, we find that the uniform coupling profile is not required to achieve HOEPs in this system - a feature that implies the emergence of HOEPs from disorder and provides resilience against some fabrication errors. Our results are confirmed by using full-wave simulations based on Maxwell's equation in realistic optical material systems.
\end{abstract}

DOI: 10.1103/PhysRevLett.125.203602

Introduction.-The notion of non-Hermitian photonics [1-4], together with its central concept of exceptional points (EPs) [5], has attracted considerable attention in the past few years. At an EP, two or more eigenvalues and their corresponding eigenvectors (i.e., eigenstates) coalesce. As a result, the dimensionality of the eigenspace is reduced. An interesting feature of systems operating at EPs is their strong (nonlinear) response to small perturbation [6,7]. More specifically, for an EP of order $N$ formed by the coalescence of $N$ different eigenvalues and associated eigenstates, the response scales as $\sqrt[N]{\epsilon}$, where $\epsilon$ is the perturbation strength. Clearly, for $N>1$, this response "amplifies" weak effects, which could be potentially useful for sensing applications [8-11], wireless energy transfer [12], and enhancing light-matter interactions [13]. However, current schemes for achieving this require the implementation of complex networks and tuning of a considerable number of design parameters [10,14-17].

In this Letter, we introduce a general approach for constructing tight-binding networks supporting higherorder exceptional points (HOEPs) out of arrangements that support only lower-order EPs. As an example, we apply our scheme for constructing photonic networks with HOEPs based on judicious engineering of the gain-loss profile and coupling coefficients in both real and synthetic dimensions. More specifically, this strategy allows us to start with a parity-time $(P T)$ symmetric arrangement having an $N$ th-order EP and modify it to obtain a $2 N$ th-order EP in a straightforward fashion. Importantly, the resultant configuration requires tuning a small number of design parameters than those of conventional $P T$ symmetric arrays with the same EP order and hence is more robust against fabrication errors and experimental uncertainties.

General procedure for doubling the order of EPs.-The proposed approach described in this section can be summarized as follows: the introduction of unidirectional coupling between two subsystems having identical EPs of order $N$ can lead to the fusion of these EPs into a single EP with order $2 N$. As we will see, this does not occur automatically but rather requires a careful engineering of the unidirectional coupling profile. To this end, we start with two different discrete systems described by two matrix Hamiltonians $H_{a}$ and $H_{b}$, each with dimension $N \times N$ and exhibiting an EP of order $N$. Moreover, we assume that both EPs correspond to the same eigenvalues. We will denote the eigenvalues and eigenvectors at the EPs by $(\mu, \vec{a})$ and $(\mu, \vec{b})$, respectively, where the $N$-dimensional column vectors $\vec{a}$ and $\vec{b}$ are the exceptional eigenvectors (i.e., the eigenvectors coalescing at the EP) of $H_{a}$ and $H_{b}$. Let us now consider the following mathematical construction:

$$
\mathcal{H}=\left[\begin{array}{cc}
H_{a} & \hat{0} \\
K & H_{b}
\end{array}\right],
$$

where $K$ is an $N \times N$ matrix, and $\hat{0}$ is an $N \times N$ null matrix. Note that $\mathcal{H}$ has a dimension $2 N \times 2 N$. The eigenvalues and eigenvectors of $\mathcal{H}$ can be written as $\left\{\lambda, \vec{c} \equiv\left[\begin{array}{l}\vec{c}_{1} \\ \vec{c}_{2}\end{array}\right]\right.$, and $\vec{c}_{1,2}$ are each $N$-dimensional column vectors (thus the column vector $\vec{c}$ has a dimension of $2 N)$. The eigenvalue problem $\lambda I-\mathcal{H}(I$ is the $N \times N$ identity matrix) can be then rearranged into 


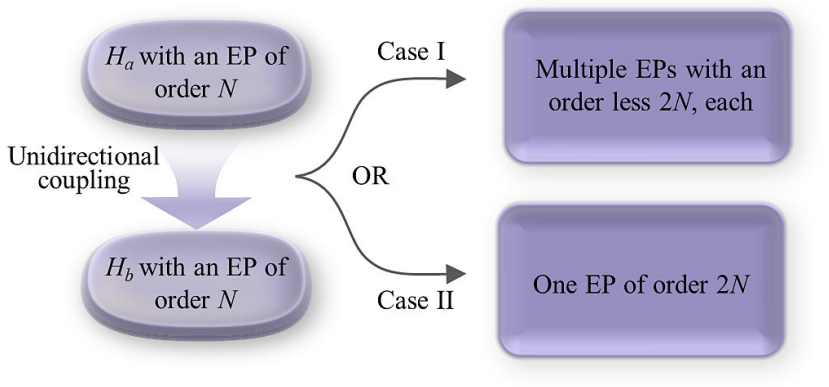

FIG. 1. By introducing a unidirectional coupling between two different Hamiltonians $H_{a}$ and $H_{b}$, each of which exhibits an EP of order $N$, the combined system in general has $M$ EPs, each of which is of order $<2 N$. As described in the text, under certain conditions, $M=1$, the system has only one EP with order $2 N$.

$$
\begin{aligned}
& \left(\lambda I-H_{a}\right) \vec{c}_{1}=0, \\
& \left(\lambda I-H_{b}\right) \vec{c}_{2}=K \vec{c}_{1} .
\end{aligned}
$$

Equation (2a) admits two possible solutions: (i) a nontrivial solution with an eigenvalue $\lambda=\mu$ and an eigenvector $\vec{c}_{1}=\vec{a}$ and (ii) a trivial one with $\vec{c}_{1}=\overrightarrow{0}$. Let us first consider the trivial solution. By substituting $\vec{c}_{1}=\overrightarrow{0}$ in Eq. (2b), we obtain $\left(\lambda I-H_{b}\right) \vec{c}_{2}=0$, which has the solution $\lambda=\mu$ and $\vec{c}_{2}=\vec{b}$. On the other hand, if we consider the nontrivial solution of Eq. (2a), we obtain $\left(\mu I-H_{b}\right) \vec{c}_{2}=K \vec{a}$. Since $\operatorname{rank}\left(\mu I-H_{b}\right)<N$, the last system of equations may be undetermined (i.e., it exhibits several solutions) or inconsistent (i.e., it has no solution), depending on the vector $K \vec{a}$. In the former case, the matrix $\mathcal{H}$ has, in general, $M$ eigenvectors that correspond to the eigenvalue $\mu$, where the actual value of $M$ depends on the system's details. The latter scenario is more interesting: it dictates that $\mathcal{H}$ has only one eigenvector $\vec{c}=[\overrightarrow{0}] \vec{b}]$ corresponding to the eigenvalue $\mu$; i.e., the spectrum of $\mathcal{H}$ has an EP of order $2 N$. These results are summarized in Fig. 1.

Having introduced the general framework, we now consider the following special case where $H_{a}=H_{b} \equiv H$ are discrete $P T$ symmetric Hamiltonians with an eigenvector $\vec{a}=\vec{b}=\vec{v}, \quad K \equiv \operatorname{diag}\left(k_{1}, k_{2}, \ldots, k_{N}\right)$ is a diagonal matrix whose elements represent a unidirectional coupling from the different elements of $H_{a}$ to the corresponding elements in $H_{b}$, as shown in Fig. 2. Without any loss of generality, we also assume that $\mu=0$. Under these conditions, Eq. (2b) reduces to $H \vec{c}_{2}=-K \vec{v}$. If this latter equation has one solution, then the spectrum of the combined system will contain two EPs of order $N$ each. On the other hand, if there is no solution, the spectrum will consist of only one EP of order $2 N$. To investigate these two situations in more detail, we express $H$ in its Jordan canonical form as defined by the similarity transformation $H^{J}=S H S^{-1}$, with $S$ being the mapping matrix. The explicit form of $H^{J}$ is

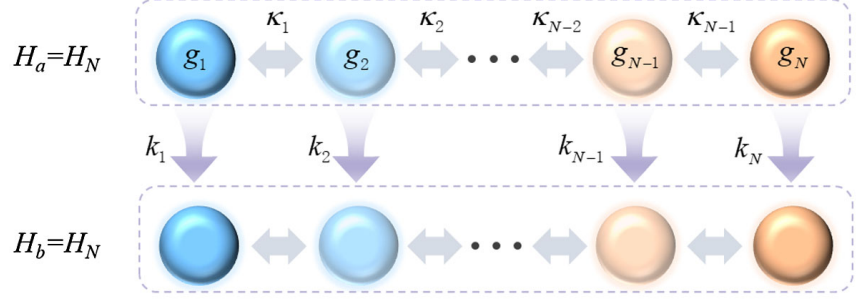

FIG. 2. A schematic of two identical copies of a $P T$ symmetric $J_{x}$ array. Each lattice has an EP of order $N$. By introducing disordered unidirectional coupling $k$ 's, the combined array can exhibit an EP of order $2 N$.

$$
H^{J}=\left[\begin{array}{cccccc}
0 & 1 & 0 & \ldots & 0 & 0 \\
0 & 0 & 1 & \ldots & 0 & 0 \\
\vdots & \vdots & \vdots & \ddots & \vdots & \vdots \\
0 & 0 & 0 & \ldots & 0 & 1 \\
0 & 0 & 0 & \ldots & 0 & 0
\end{array}\right], \quad \vec{v}^{J}=\left[\begin{array}{c}
1 \\
0 \\
\vdots \\
0
\end{array}\right]
$$

In these bases, $K^{J}=S K S^{-1}, \vec{c}_{2}^{J}=S \vec{c}_{2}$, and $\vec{v}^{J}=S \vec{v}$. Accordingly,

$$
H^{J} \vec{c}_{2}^{J}=-K^{J} \vec{v}^{J}
$$

If the diagonal matrix elements of $K$ are identical and equal to $k$, the combined structure will respect $P T$ symmetry, and we obtain $K^{J}=K=k I$. It follows that Eq. (4) is undetermined with a family of solutions given by $\vec{c}_{2}^{J}=$ $[x,-k, 0, \ldots, 0]^{T}$, where $x$ is a free parameter and the superscript $T$ indicates transpose. Clearly, the spectrum of $\mathcal{H}$ in this case does not contain an EP of order $2 N$. A more general argument applies when $[S, K]=0$. If, however, one introduces a disorder to the coupling matrix $K$ (i.e., introducing random perturbations to the coupling coefficient $k$ ), such that the $N$ th element of the vector $K^{J} \vec{v}^{J}$ is not zero, then Eq. (4) is inconsistent and has no solution, and thus $\mathcal{H}$ would contain an EP of order $2 N$. In this case, we will denote the Hamiltonian as $\mathcal{H}_{2 N}$.

Application to $P T J_{x}$ arrays: In order to illustrate the power of the scheme presented above, we demonstrate its application to $J_{x}$ photonic array [18-20] that possesses $P T$ symmetry [14]. A tight-binding network that realizes an EP of order $N$ using $P T J_{x}$ arrays will consist of $N$ sites, and its Hamiltonian $H_{N}$ is given by [14,21] (see Supplemental Material [22] for more details)

$$
H_{N}=\left[\begin{array}{ccccc}
i g_{1} & \kappa_{1} & \ldots & 0 & 0 \\
& & \vdots & & \\
\ldots & \kappa_{n-1} & i g_{n} & \kappa_{n} & \ldots \\
& & \vdots & & \\
0 & 0 & \ldots & \kappa_{N-1} & i g_{N}
\end{array}\right]
$$


In the above equation, $g_{n}=(2 n-N-1) \gamma$ and $\kappa_{n}=$ $\sqrt{n(N-n)} \gamma$ with $n=1,2, \ldots, N$, and $\gamma$ is a nonHermitian parameter (in optics, it represents gain or loss depending on its sign). Obviously, an implementation of an EP of order $2 \mathrm{~N}$ can be directly obtained by scaling the above system according to $N \rightarrow 2 N$ to obtain $H_{2 N}$. This, however, introduces more nonuniformity in the coupling and gain-loss profiles, which poses practical limitations on realizing these arrangements. On the other hand, our scheme relaxes some of these constraints by enabling the construction of an EP of order $2 N$ out of two exact copies of $H_{N}$ according to Eq. (1) by substituting $H_{a}=H_{b}=H_{N}$, as demonstrated schematically in Fig. 2 . In what follows, we take $K$ to a be a diagonal matrix and we will show that this choice can lead to an EP of order $2 N$. It is worth highlighting that both $H_{2 N}$ and $\mathcal{H}_{2 N}$ are connected via a similarity transformation. This can be demonstrated by noting that both $H_{2 N}$ and $\mathcal{H}_{2 N}$ have the same Jordan form, which we denote by $H_{2 N}^{J}$, i.e., $R^{-1} H_{2 N} R=H_{2 N}^{J}=$ $S^{-1} \mathcal{H}_{2 N} S$ for some mapping matrices $R$ and $S$. This connection can be also expressed in terms of discrete supersymmetry [27,28]: by setting $A=R S, B=\mathcal{H}_{2 N}(R S)^{-1}$, we find $H_{2 N}=A B$ and $\mathcal{H}_{2 N}=B A$.

Before we proceed further, we would like to mention that a few studies have presented different routes for implementing higher-order EPs. For instance, Nada et al. proposed a scheme that leads to high-order EP without relying on gain-loss distribution [17]. Our Letter goes beyond previous studies by introducing a straightforward way of modifying an already implemented system that has $N$ th-order EP to double the order of the EP to $2 N$, with the minimal number of additional design parameters, and by showing for the first time that higher-order EPs can arise due to disorder, which completely goes against the conventional wisdom in the field.

Implementation of $\mathcal{H}_{2 N}$ using synthetic dimensions.-In principle, the structure shown in Fig. 2 can be implemented by using $2 N$ resonators together with optical isolators to introduce the unidirectional coupling. This, however, does not provide any advantage in terms of scalability or fabrication. Alternatively, we explore a different route that relies on synthetic dimensions [29-33]. Particularly, we consider a $P T$ symmetric $J_{x}$ array made of $N$ microring resonators. Time reversal symmetry implies that each resonator supports two traveling waves at each resonant frequency, clockwise $(\mathrm{CW})$ and counterclockwise $(\mathrm{CCW})$. Taken together, these $2 \mathrm{~N}$ modes can act as bases for implementing an EP of order 2N. Figure 3(a) illustrates one possible realization of the above photonic network when $N=2$. The unidirectional coupling here is introduced via an evanescently coupled waveguide with an end mirror - a geometry that was recently proposed for building robust EP sensors [34], amplifiers [35], and directional absorbers [36]. Figure 3(b) depicts the equivalent arrangement in real space. Interestingly, if a mirror is introduced on each waveguide, the system will not exhibit a HOEP.

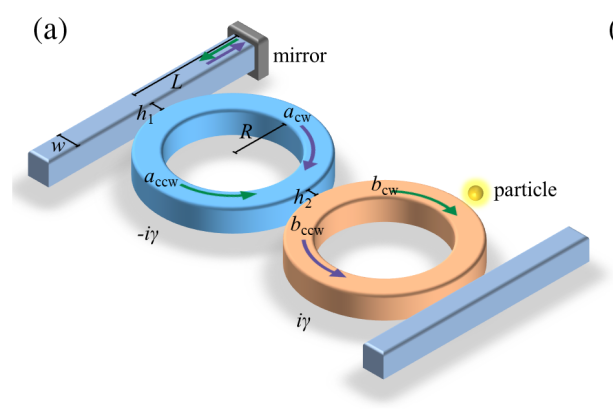

(b)

FIG. 3. (a) A $P T$ symmetric system consisting of two coupled microring resonators having equal loss $(-i \gamma)$ and gain $(i \gamma)$ implements an EP of order 2. By introducing an evanescent coupling between any of the rings (the lossy ring here) and a waveguide with an end mirror, the system realizes the Hamiltonian $\mathcal{H}_{4}$ of Eq. (6). The nanoparticle close to the gain ring is used as a small perturbation to confirm that the combined system indeed has an EP of order 4 in the simulations. (b) Equivalent real space implementation to the system in (a), detailing the gain-loss distribution and the coupling profile between various $\mathrm{CW}$ and CCW components.

In other words, the formation of the HOEP in this structure can be achieved only by breaking the $P T$ symmetry-a rather remarkable observation.

Applications in enhanced light-matter interaction: Before we present a concrete design for the above structure with realistic material systems, we first confirm that it can be indeed used to enhance light-matter interaction. As a prototype example, we focus again on the implementation of $\mathcal{H}_{4}$ as shown in Fig. 3 and we assume that a nanoparticle exists in the near field region of one of the resonators as shown in Fig. 3. By assuming that the nanoparticle introduces a perturbation strength $\epsilon$, it follows from the perturbation theory of nonHermitian operators [6,7] that an eigenfrequency splitting scaling $\sqrt[4]{\epsilon}$ should take place. To confirm that this is indeed the case for our structure, we consider its Hamiltonian as derived by using temporal coupled mode theory [37], which takes the form $\mathcal{H}_{4}^{\prime}=\mathcal{H}_{4}+H_{p}$, with

$$
\begin{aligned}
\mathcal{H}_{4} & =\left[\begin{array}{cccc}
\omega_{0}-i \gamma & \gamma & 0 & 0 \\
\gamma & \omega_{0}+i \gamma & 0 & 0 \\
k & 0 & \omega_{0}-i \gamma & \gamma \\
0 & 0 & \gamma & \omega_{0}+i \gamma
\end{array}\right], \\
H_{p} & =\left[\begin{array}{llll}
0 & 0 & 0 & 0 \\
0 & \epsilon & 0 & \epsilon \\
0 & 0 & 0 & 0 \\
0 & \epsilon & 0 & \epsilon
\end{array}\right] .
\end{aligned}
$$

Here $\omega_{0}$ is the resonant frequency of each mode, $\pm \gamma$ is the gain-loss coefficients of the modes in the gain and loss resonators, which is also taken to be identical to the coupling coefficient between the two resonators in order to implement a 
$P T$ symmetric dimer (when $k=0$ ). Additionally, $H_{p}$ is the perturbation Hamiltonian, and $\epsilon=|\epsilon| \exp \left(i \phi_{\epsilon}\right)$. We remark that the above Hamiltonian is expressed in the bases $\left[a_{\mathrm{CW}}, b_{\mathrm{CCW}}, a_{\mathrm{CCW}}, b_{\mathrm{CW}}\right]^{T}$ (see Fig. 3). When $\epsilon=0$, the above system has an EP4 with an eigenvalue $\omega=\omega_{0}$. Assuming a small perturbation $|\epsilon| \ll|\gamma|,|k|$, we obtain the following asymptotic expression (see the Supplemental Material [22]) for the eigenvalues $\omega_{n}$ :

$$
\omega_{n}-\omega_{0}=\chi_{n} \sqrt[4]{|\epsilon|}+\mathcal{O}\left(\sqrt[4]{|\epsilon|^{3}}\right)
$$

where $\chi_{n}=i^{n-1} \sqrt[4]{\exp \left(i \phi_{\epsilon}\right) k \gamma^{2}}$, and $n=1,2,3$, 4. In other words, by just evanescently coupling the resonators to waveguides and terminating one waveguide with a mirror, the eigenvalue splitting changes from $\sqrt{|\epsilon|}$ to $\sqrt[4]{|\epsilon|}$. Similarly, one can apply the same strategy to the $P T$ system that supports $\mathrm{EP}$ of order 3, which has been implemented experimentally in [10], in order to obtain a modified structure supporting an EP of order 6.

Full-wave simulations: Finally, we confirm our results by performing full-wave analysis using the finite element method (FEM) [38] for the structure of Fig. 3(a) with realistic dimensions and material systems as detailed in the Supplemental Material [22]. A full account of how to extract the relevant optical parameters numerically is described in the Supplemental Material [22]. Figures 4(a) and 4(b) present the real and imaginary values of the four eigenfrequencies of the system as a function of the perturbation induced by nanoparticles of different radii (see the Supplemental Material [22] for the relation between $|\epsilon|$ and nanoparticle radius $R_{p}$ ). The solid line represents analytical results obtained from Eq. (7), while the dots represent actual values obtained from full-wave numerical simulations using FEM. As expected, the perturbation due to the particle causes the unperturbed eigenstate to split into four different eigenmodes. From the figures, we observe good agreement between theory and simulations.

Figure 4(c) plots $\operatorname{Re}\left[\omega_{1}-\omega_{0}\right]$ as a function of $\epsilon$ using $\log$ scale. A curve fitting of the data produces the relation $\operatorname{Re}\left[\omega_{1}-\omega_{0}\right]=A \epsilon^{0.24}$, which corresponds to a line with a slope 0.24 on the log scale, i.e., only $4 \%$ deviation from the ideal value of 0.25 expected for an ideal EP of order 4 . Further discussions of the numerical results are presented in the Supplemental Material [22].

Conclusions. - In summary, we have introduced a new, generic approach for constructing tight-binding Hamiltonians with HOEPs out of initial arrangements with lower-order EPs. As an illustrative example, we have demonstrated the detailed application of our scheme to $P T$ symmetric $J_{x}$ arrays. By focusing on the $P T$ symmetric dimer and utilizing the concept of synthetic dimensions in multimodal systems, we have presented an elegant and more robust (compared to conventional configurations) implementation of an optical ring dimer that exhibits a fourth-order EP. We should note here that many interesting
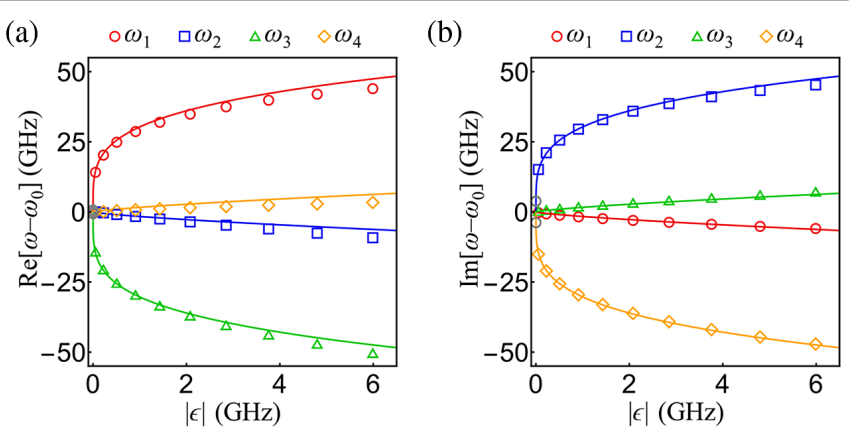

(c)

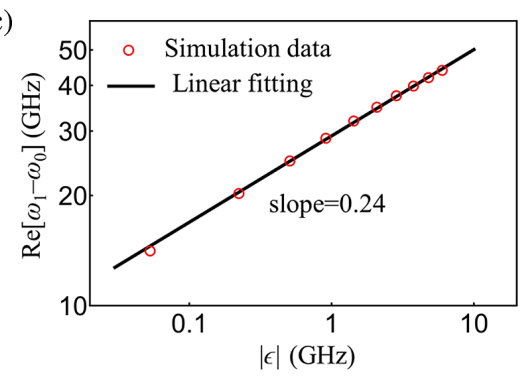

FIG. 4. (a) Real and (b) imaginary splitting of the eigenfrequency of the system in Fig. 3(a) as a result of a perturbation $|\epsilon|$ (see the Supplemental Material [22]) introduced by a nanoparticle. Dots represent the results obtained from full-wave FEM simulations for the ring resonator parameters given in the Supplemental Material [22], while the solid black line is a plot of the analytical formula of Eq. (7). (c) Log scale plot of the real frequency shift $\operatorname{Re}\left[\omega_{1}-\omega_{0}\right]$ as a function of $|\epsilon|$. The slope of the linear fit is $\sim 0.24$, confirming that the system is operating in the vicinity of an EP of order 4.

features of EPs, such as a system's response to perturbations as in sensors, stem from the order of EPs: the higher the better, regardless of whether it is an odd- or even-order EP. Mathematically speaking, there is no bound on the order of EP that can be engineered using our approach. Limitations originate from the maturity and scalability of the fabrication process of the underlined physical platform. For example, in electronics, where fabrication is highly scalable and fabrication errors are minimal, one can construct an EP of any even order for telemetry and wireless energy transfer. Scalability in photonics has always been a critical problem despite decent progress in the past few years, and, in general, building photonic systems with HOEPs is challenging (i.e., none beyond order 3 yet). Our approach provides a methodological approach and a realistic route to build photonic systems with HOEPs, alleviating some of the difficulties encountered. This, in turn, may have an impact on building better sensing devices (though this topic is still subject to debate, see, for instance, [39-44]), as well as photonic components with enhanced nonlinear interactions.

R. E. acknowledges fruitful discussions with J. Wiersig. R.E. acknowledges support from ARO (Grant No. W911NF-17-1-0481), NSF (Grant No. ECCS 1807552), the Max Planck Institute for the Physics of 
Complex Systems, and the Henes Center for Quantum Phenomena at Michigan Technological University. S. K. O acknowledges support from ARO (Grant No. W911NF-181-0043), NSF (Grant No. ECCS 1807485), and AFOSR (Grant No. FA9550-18-1-0235).

*Corresponding author. ganainy@mtu.edu

[1] R. El-Ganainy, K. G. Makris, M. Khajavikhan, Z.H. Musslimani, S. Rotter, and D. N. Christodoulides, Nat. Phys. 14, 11 (2018).

[2] L. Feng, R. El-Ganainy, and L. Ge, Nat. Photonics 11, 752 (2017).

[3] R. El-Ganainy, M. Khajavikhan, D. N. Christodoulides, and S. K. Ozdemir, Commun. Phys. 2, 37 (2019).

[4] Ş. K. Özdemir, S. Rotter, F. Nori, and L. Yang, Nat. Mater. 18, 783 (2019).

[5] M.-A. Miri and A. Alù, Science 363, eaar7709 (2019).

[6] T. Kato, Perturbation Theory for Linear Operators (Springer, New York, 1995).

[7] Y. Ma and A. Edelman, Linear Algebra Appl. 273, 45 (1998).

[8] J. Wiersig, Phys. Rev. Lett. 112, 203901 (2014).

[9] J. Wiersig, Phys. Rev. A 93, 033809 (2016).

[10] H. Hodaei, A. U. Hassan, S. Wittek, H. Garcia-Gracia, R. El-Ganainy, D. N. Christodoulides, and M. Khajavikhan, Nature (London) 548, 187 (2017).

[11] W. Chen, Ş. K. Özdemir, G. Zhao, J. Wiersig, and L. Yang, Nature (London) 548, 192 (2017).

[12] S. Assawaworrarit, X. Yu, and S. Fan, Nature (London) 546, 387 (2017).

[13] A. Pick, B. Zhen, O. D. Miller, C. W. Hsu, F. Hernandez, A. W. Rodriguez, M. Soljačić, and S. G. Johnson, Opt. Express 25, 12325 (2017).

[14] M. H. Teimourpour, R. El-Ganainy, A. Eisfeld, A. Szameit, and D. N. Christodoulides, Phys. Rev. A 90, 053817 (2014).

[15] M. H. Teimourpour, Q. Zhong, M. Khajavikhan, and R. El-Ganainy, Higher order exceptional points in discrete photonics platforms, in Parity-Time Symmetry and Its Applications, edited by D. Christodoulides and J. Yang (Springer, Singapore, 2018), pp. 261-275.

[16] B. Peng, Ş. K. Özdemir, F. Lei, F. Monifi, M. Gianfreda, G. L. Long, S. Fan, F. Nori, C. M. Bender, and L. Yang, Nat. Phys. 10, 394 (2014).

[17] M. Y. Nada, M. A. K. Othman, and F. Capolino, Phys. Rev. B 96, 184304 (2017).

[18] M. Christandl, N. Datta, A. Ekert, and A. J. Landahl, Phys. Rev. Lett. 92, 187902 (2004).

[19] A. Perez-Leija, R. Keil, A. Kay, H. Moya-Cessa, S. Nolte, L.-C. Kwek, B. M. Rodríguez-Lara, A. Szameit, and D. N. Christodoulides, Phys. Rev. A 87, 012309 (2013).

[20] M. H. Teimourpour, A. Rahman, K. Srinivasan, and R. ElGanainy, Phys. Rev. Applied 7, 014015 (2017).
[21] Q. Zhong, D. N. Christodoulides, M. Khajavikhan, K. G. Makris, and R. El-Ganainy, Phys. Rev. A 97, 020105(R) (2018)

[22] See Supplemental Material at http://link.aps.org/ supplemental/10.1103/PhysRevLett.125.203602 for nonHermitian $J_{x}$ photonic array, eigenvalues of Hamiltonian under perturbation, geometric, material, and optical parameters in simulation, perturbation coefficient due to the nanoparticle, modal profile, and further comments on the numerical results, which includes Refs. [23-26].

[23] A. Perez-Leija, R. Keil, H. Moya-Cessa, A. Szameit, and D. N. Christodoulides, Phys. Rev. A 87, 022303 (2013).

[24] R. El-Ganainy, A. Eisfeld, M. Levy, and D. N. Christodoulides, Appl. Phys. Lett. 103, 161105 (2013).

[25] R. J. Chapman, M. Santandrea, Z. Huang, G. Corrielli, A. Crespi, M.-H. Yung, R. Osellame, and A. Peruzzo, Nat. Commun. 7, 11339 (2016).

[26] J. Zhu, Ş. K. Özdemir, Y.-F. Xiao, L. Li, L. He, D.-R. Chen, and L. Yang, Nat. Photonics 4, 46 (2010).

[27] M.-A. Miri, M. Heinrich, R. El-Ganainy, and D. N. Christodoulides, Phys. Rev. Lett. 110, 233902 (2013).

[28] R. El-Ganainy, L. Ge, M. Khajavikhan, and D. N. Christodoulides, Phys. Rev. A 92, 033818 (2015).

[29] A. Celi, P. Massignan, J. Ruseckas, N. Goldman, I. B. Spielman, G. Juzeliunas, and M. Lewenstein, Phys. Rev. Lett. 112, 043001 (2014).

[30] L. F. Livi, G. Cappellini, M. Diem, L. Franchi, C. Clivati, M. Frittelli, F. Levi, D. Calonico, J. Catani, M. Inguscio, and L. Fallani, Phys. Rev. Lett. 117, 220401 (2016).

[31] T. Ozawa, H. M. Price, N. Goldman, O. Zilberberg, and I. Carusotto, Phys. Rev. A 93, 043827 (2016).

[32] E. Lustig, S. Weimann, Y. Plotnik, Y. Lumer, M. A. Bandres, A. Szameit, and M. Segev, Nature (London) 567, 356 (2019).

[33] L. Yuan, Q. Lin, M. Xiao, and S. Fan, Optica 5, 1396 (2018).

[34] Q. Zhong, J. Ren, M. Khajavikhan, D. N. Christodoulides, Ş. K. Özdemir, and R. El-Ganainy, Phys. Rev. Lett. 122, 153902 (2019).

[35] Q. Zhong, S. K. Ozdemir, A. Eisfeld, A. Metelmann, and R. El-Ganainy, Phys. Rev. Applied 13, 014070 (2020).

[36] Q. Zhong, S. Nelson, Ş. K. Özdemir, and R. El-Ganainy, Opt. Lett. 44, 5242 (2019).

[37] W. Chen, J. Zhang, B. Peng, Ş. K. Özdemir, X. Fan, and L. Yang, Photonics Res. 6, A23 (2018).

[38] The simulations were performed using COMSOL software package.

[39] W. Langbein, Phys. Rev. A 98, 023805 (2018).

[40] M. Zhang, W. Sweeney, C. W. Hsu, L. Yang, A. D. Stone, and L. Jiang, Phys. Rev. Lett. 123, 180501 (2019).

[41] H.-K. Lau and A. A. Clerk, Nat. Commun. 9, 4320 (2018)

[42] J. Wiersig, Nat. Commun. 11, 2454 (2020).

[43] J. Wiersig, Phys. Rev. A 101, 053846 (2020).

[44] J. Wiersig, Photonics Res. 8, 1457 (2020). 